\title{
HIGH VELOCITY OUTFLOWS IN IRAS 17423-1755
}

\author{
A. RIERA \\ Departament d'Astronomia i Meteorologia, Universitat de Barcelona, Av. Diagonal \\ 647, E-08028 Barcelona (Spain) and Universitat Politcnica de Catalunya \\ P. GARCíA-LARIO \\ LAEFF - Estación de Villafranca del Castillo. Apdo. 50727. E-28080 Madrid (Spain) \\ A. MANCHADO \\ Instituto de Astrofísica de Canarias. E-38200, La Laguna, Tenerife (Spain) \\ and \\ S.R. POTTASCH
}

Kapteyn Laboratorium, Postbus 800. NL-9700, AV Groningen (The Netherlands)

\begin{abstract}
IRAS 17423-1755 is a new transition object between the postAGB phase and the planetary nebula (PN) stage included in an observational program of IRAS sources with infrared colours similar to those of PNe. We have taken $\mathrm{B}, \mathrm{V}, \mathrm{R}, \mathrm{I}, \mathrm{Z}, \mathrm{H} \alpha$ and [OIII] CCD images at the $1 \mathrm{~m} \mathrm{JKT}$ (Roque de los Muchachos, La Palma), where a clearly marked bipolar structure with a total extension of about 10 " can be seen, and long slit (low and high resolution) optical spectra at the $4.2 \mathrm{~m}$ WHT, 2.5m INT (RM, La Palma) and ESO 1.5m (La Silla) telescopes.

The spectrum of the core corresponds to a B star with strong emission lines of HI Balmer and Paschen series, FeII, [FeII], OI, CaII, and [CaII], sharing characteristics with $B Q[]$ stars, which are stars in the post-AGB phase, while on the other hand, proto-PNe usually show a similar morphology (He2-123, M2-9 or M1-16).

The study of the OI $\lambda 8446 \AA$ emission reveals that it is due to starlight excitation and/or Ly $\beta$ fluorescence. The detection of strong CaII triplet lines implies the existence of a predominantly neutral HI region (i.e. a cool circumstellar disk) with densities from $10^{9}$ to $10^{15} \mathrm{~cm}^{-3}$. A rich [FeII] emission line spectrum is observed, which is probably excited by collisions in a gas with $\mathrm{n}_{e} \sim 10^{5}-10^{6} \mathrm{~cm}^{-3}$.

The most remarkable kinematic features are the widening of the assymetric $\mathrm{H} \alpha$ profile at the core, the tilt in velocity of the lobes and the high-velocity feature (jet) connecting the core and the redshifted lobe. Line profiles observed in the lobes are characterized by large radial velocities $\left(\gtrsim 425 \mathrm{~km} \mathrm{~s}^{-1}\right)$, extraordinary line widths and double-peaked profiles, indicating that the emission arises from the cooling region behind a bow-shock, with a shock velocity of $\sim 260 \mathrm{~km} \mathrm{~s}^{-1}$. The jet decelerates as it moves away from the central source, its velocity decreasing from $920 \mathrm{~km} \mathrm{~s}^{-1}$ to $750 \mathrm{~km} \mathrm{~s}^{-1}$. From the high values of the [NII] $(6548+6584) / \mathrm{H} \alpha$ ratios $(\sim 4)$ measured in the lobes and in the jet, we deduce that the outflowing material is nitrogen enriched gas of stellar origin.

Theoretical models predict the formation of bow-shocks at the end of highly collimated outflows, when a high velocity wind with an axial jet interacts with the surrounding material. It is argued that assymetric mass loss in the post-AGB phase could be the responsible of the formation of bipolar structures, as an alternative to the binary hypothesis.
\end{abstract}

